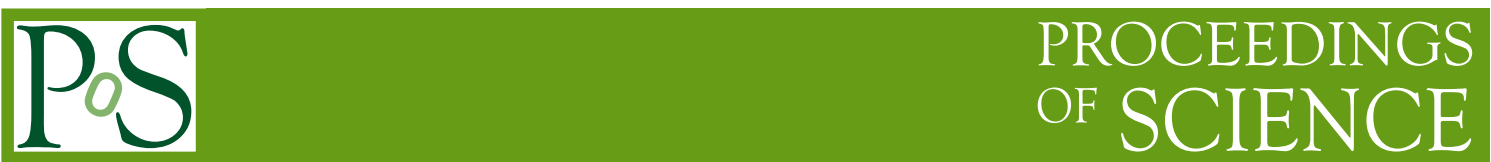

\title{
Non-commutative geometry and matrix models
}

\author{
Harold Steinacker* \\ Fakultät für Physik, Universität Wien \\ Boltzmanngasse 5, A-1090 Wien, Austria \\ E-mail: harold.steinacker@univie.ac.at
}

These notes provide an introduction to the noncommutative matrix geometry which arises within matrix models of Yang-Mills type. Starting from basic examples of compact fuzzy spaces, a general notion of embedded noncommutative spaces (branes) is formulated, and their effective Riemannian geometry is elaborated. This class of configurations is preserved under small deformations, and is therefore appropriate for matrix models. A realization of generic 4-dimensional geometries is sketched, and the relation with spectral geometry and with noncommutative gauge theory is explained. In a second part, dynamical aspects of these matrix geometries are considered. The one-loop effective action for the maximally supersymmetric IKKT or IIB matrix model is discussed, which is well-behaved on 4-dimensional branes.

3rd Quantum Gravity and Quantum Geometry School

February 28 - March 13, 2011

Zakopane, Poland

\footnotetext{
* Speaker.
} 


\section{Introduction}

Our basic notion of space and time go back to Einstein. Space-time is described in terms of a pseudo-Riemannian manifold, whose dynamical metric describes gravity through the Einstein equations. This concept also provides a basis for quantum field theory, where the metric is usually assumed to be flat, focusing on the short-distance aspects of the fields living on space-time.

Despite the great success of both general relativity and quantum field theory, there are good reasons why we should question these classical notions of space and time. The basic reason is that nature is governed by quantum mechanics. Quantum mechanics is fundamentally different from classical physics, and the superposition principle rules out a description in terms of sharply defined classical objects and states. Since general relativity (GR) couples matter with geometry, a superposition of matter entails also a superposition of geometries. We are thus forced to look for a consistent quantum theory of fields and geometry, hence of gravity.

Constructing a quantum theory of gravity is clearly a difficult task. Not only is general relativity not renormalizable, there are arguments which suggest that the classical geometric concepts are inappropriate at very short distances. A simple folklore argument goes as follows: localizing an object at a scale $\Delta x$ in quantum mechanics requires to invoke wave-numbers $k \sim \frac{1}{\Delta x}$, and thus an energy of order $E=\hbar k \sim \frac{\hbar}{\Delta x}$. Now in general relativity, a localized energy $E$ defines a length scale given by the corresponding Schwarzschild radius $R_{\text {Schwarzschild }} \sim G E \geq \frac{\hbar G}{\Delta x}$. Since observations inside trapped surfaces do not make sense, one should require $(\Delta x) \geq R_{\text {Schwarzschild }} \geq \frac{\hbar G}{\Delta x}$, hence $(\Delta x)^{2} \geq \hbar G=L_{P l}^{2}$. Of course the argument is over-simplistic, however a refined argument [1] suggests that quantum mechanics combined with GR implies uncertainly relations for the space-time coordinates at the Planck scale. Even if one does not want to take such "derivations" too serious, there is common consensus that space-time should become fuzzy or foam-like at the Planck scale. Canonical or loop quantum gravity indeed leads to an area quantization at the Planck scale, and in string theory something similar is expected to happen [2].

The short-distance aspects of space-time are problematic also within quantum field theory (QFT), leading to the well-known UV divergences. They can be handled in renormalizable QFT's, but imply that some low-energy properties of the models are very sensitive to the short-distance physics. This leads to serious fine-tuning problems e.g. for the mass of scalar (Higgs!) fields, which strongly suggests new physics at short distances unless one is willing to accept an anthropic point of view. Taking into account also gravity leads to even more serious fine-tuning problems, notably the notorious cosmological constant problem. The point is that vacuum fluctuations in quantum field theory couple to the background metric, which leads to an induced gravitational action, in particular to an induced cosmological constant. Lacking any natural subtraction scheme for these terms, these contributions are strongly UV divergent, or very sensitive to the UV details of the model. No convincing solution to this problem has been found, which should arise in all approaches based on general relativity including loop quantum gravity and string theory.

Given all these difficulties, we will discuss a radically different approach here. Since the notions of space-time and geometry were argued to make sense only at macroscopic scales, the basic degrees of freedom in a fundamental quantum theory may be very different from the macroscopic ones, while space-time and geometry "emerge" in some semi-classical sense. This idea is of course not new, and there are many models where some effective metric emerges in composite systems. 
However, we need a model which leads to a universal dynamical metric coupling to a (near-) realistic quantum field theory, simple enough to admit an analytic understanding.

In these notes, we will discuss certain specific matrix models of Yang-Mills type, which seem to realize this idea of emergent geometry and gravity in a remarkably simple way. These models have been put forward in string theory [3,4], and may provide a description for the quantum structure of space-time and geometry. The beauty lies in the simplicity of these models, whose structure is

$$
S_{Y M}=\operatorname{Tr}\left[X^{a}, X^{b}\right]\left[X^{a^{\prime}}, X^{b^{\prime}}\right] g_{a a^{\prime}} g_{b b^{\prime}}+\text { fermions. }
$$

Here $X^{a}, a=1, \ldots, D$ are a set of hermitian matrices, and we focus on the case of Euclidean signature $g_{a b}=\delta_{a b}$ in this article. No notion of differential geometry or space-time is used in this action. These geometrical structures arise in terms of solutions and fluctuations of the models. The aim of this article is to clarify the scope and the mathematical description of this matrix geometry.

Simple examples of such matrix geometries, notably the fuzzy sphere $S_{N}^{2}$ or more general quantized homogeneous spaces including the Moyal-Weyl quantum plane $\mathbb{R}_{\theta}^{2 n}$, have been studied in great detail. However to describe the general geometries required for gravity, one cannot rely on their special group-theoretical structures. The key is to consider generic quantizations of submanifolds or embedded noncommutative $(N C)$ branes $\mathscr{M} \subset \mathbb{R}^{D}$ in Yang-Mills matrix models [5]. This provides a sufficiently large class of matrix geometries to describe realistic space-times. Their effective geometry is easy to understand in the "semi-classical limit", where commutators are replaced by Poisson brackets. $\mathscr{M}$ then inherits the pull-back metric $g_{\mu v}$ of $\mathbb{R}^{D}$, which combines with the Poisson (or symplectic) structure $\theta^{\mu v}(x)$ to form an effective metric $G_{\mu v}(x)$. Our task is then to elaborate the resulting physics of these models, and to identify the necessary mathematical structures to understand them.

The aim of these notes is to provide a basic understanding of matrix geometry and its mathematical description, and to explain the physical relevance of matrix models. We first recall in detail some examples of matrix geometries with special symmetries. This includes well-known examples such as the fuzzy sphere, fuzzy tori, cylinders, and the quantum plane. We then explain how to extract the geometry without relying on particular symmetries. The spectral geometry of the canonical Laplace operator is discussed, and compared with a semi-classical analysis. An effort is made to illustrate the scope and generality of matrix geometry. The remarkable relation between matrix geometry and noncommutative gauge theory $[6,7,8]$ is also discussed briefly.

Our focus on matrix geometry is justified by the good behavior of certain Yang-Mills matrix models - more precisely, of one preferred incarnation given by the IKKT model [3] - under quantization. This will be explained in section 10 . The IKKT model is singled out by supersymmetry and its (conjectured) UV finiteness on 4-dimensional backgrounds, and it may provide just the right degrees of freedom for a quantum theory of fundamental interactions. All the ingredients required for physics may emerge from the model, and there is no need to add additional structure. Our strategy is hence to study the resulting physics of these models and to identify the appropriate structures, while minimizing any mathematical assumptions or prejudices. It appears that Poisson or symplectic structures do play a central role. This is the reason why the approach presented here does not follow Connes axioms [9] for noncommutative geometry, but we will indicate some relations where appropriate. 


\section{Poisson manifolds and quantization}

We start by recalling the concept of the quantization of a Poisson manifold $(\mathscr{M},\{.,\}$.$) , refer-$ ring e.g. to [10] and references therein for more mathematical background. A Poisson structure is an anti-symmetric bracket $\{.,\}:. \mathscr{C}(\mathscr{M}) \times \mathscr{C}(\mathscr{M}) \rightarrow \mathscr{C}(\mathscr{M})$ which is a derivation in each argument and satisfies the Jacobi identity,

$$
\{f g, h\}=f\{g, h\}+g\{f, h\}, \quad\{f,\{g, h\}\}+\text { cycl. }=0 .
$$

We will usually assume that $\theta^{\mu v}=\left\{x^{\mu}, x^{v}\right\}$ is non-degenerate, thus defining a symplectic form

$$
\omega=\frac{1}{2} \theta_{\mu \nu}^{-1} d x^{\mu} d x^{\nu}
$$

in local coordinates. In particular, the dimension $\operatorname{dim} \mathscr{M}=2 n$ must then be even, and $d \omega=0$ is equivalent to the Jacobi identity.

It is sometimes useful to introduce an expansion parameter $\theta$ of dimension lengt $h^{2}$ and write

$$
\left\{x^{\mu}, x^{v}\right\}=\theta^{\mu v}(x)=\theta \theta_{0}^{\mu v}(x)
$$

where $\theta_{0}^{\mu v}$ is some fixed Poisson structure. Given a Poisson manifold, we denote as quantization map an isomorphism of vector spaces

$$
\begin{aligned}
\mathscr{I}: \quad \mathscr{C}(\mathscr{M}) & \rightarrow \mathscr{A} \subset \operatorname{Mat}(\infty, \mathbb{C}) \\
f(x) & \mapsto F
\end{aligned}
$$

which depends on the Poisson structure $\mathscr{I} \equiv \mathscr{I}_{\theta}$, and satisfies ${ }^{1}$

$$
\mathscr{I}(f g)-\mathscr{I}(f) \mathscr{I}(g) \rightarrow 0 \quad \text { and } \quad \frac{1}{\theta}(\mathscr{I}(i\{f, g\})-[\mathscr{I}(f), \mathscr{I}(g)]) \rightarrow 0 \quad \text { as } \quad \theta \rightarrow 0 .
$$

Here $\mathscr{C}(\mathscr{M})$ denotes a suitable space of functions on $\mathscr{M}$, and $\mathscr{A}$ is interpreted as quantized algebra of functions ${ }^{2}$ on $\mathscr{M}$. Such a quantization map $\mathscr{I}$ is not unique, i.e. the higher-order terms in (2.5) are not unique. Sometimes we will only require that $\mathscr{I}$ is injective after a UV-truncation to $\mathscr{C}_{\Lambda}(\mathscr{M})$ defined in terms of a Laplace operator, where $\Lambda$ is some UV cutoff. This is sufficient for physical purposes. In any case, it is clear that $\theta^{\mu v}$ - if it exists in nature - must be part of the dynamics of space-time. This will be discussed below.

The map $\mathscr{I}$ allows to define a "star" product on $\mathscr{C}(\mathscr{M})$ as the pull-back of the algebra $\mathscr{A}$,

$$
f \star g:=\mathscr{I}^{-1}(\mathscr{I}(f) \mathscr{I}(g)) .
$$

It allows to work with classical functions, hiding $\theta^{\mu v}$ in the star product. Kontsevich has shown [11] that such a quantization always exists in the sense of formal power series in $\theta$. This is a bit too weak for the present context since we deal with operator or matrix quantizations. In the case of compact symplectic spaces, existence proofs for quantization maps in the sense of operators as required here are available [10], and we will not worry about this any more. Finally, the integral over the classical space is related in the semi-classical limit to the trace over its quantization as follows

$$
\int \frac{\omega^{n}}{n !} f \sim(2 \pi)^{n} \operatorname{Tr} \mathscr{I}(f) .
$$

\footnotetext{
${ }^{1}$ The precise definition of this limiting process is non-trivial, and there are various definitions and approaches. Here we simply assume that the limit and the expansion in $\theta$ exist in some appropriate sense.

${ }^{2} \mathscr{A}$ is the algebra generated by $X^{\mu}=\mathscr{I}\left(x^{\mu}\right)$, or some subalgebra corresponding to well-behaved functions.
} 
Embedded noncommutative spaces. Now consider a Poisson manifold embedded in $\mathbb{R}^{D}$. Denoting the Cartesian coordinate functions on $\mathbb{R}^{D}$ with $x^{a}, a=1, \ldots, D$, the embedding is encoded in the maps

$$
x^{a}: \quad \mathscr{M} \hookrightarrow \mathbb{R}^{D},
$$

so that $x^{a} \in \mathscr{C}(\mathscr{M})$. Given a quantization (2.4) of the Poisson manifold $(\mathscr{M},\{.,\}$.$) , we obtain$ quantized embedding functions

$$
X^{a}:=\mathscr{I}\left(x^{a}\right) \in \mathscr{A} \subset \operatorname{Mat}(\infty, \mathbb{C})
$$

given by specific (possibly infinite-dimensional) matrices. This defines an embedded noncommutative space, or a NC brane. These provide a natural class of configurations or backgrounds for the matrix model (1.1), which sets the stage for the following considerations. The map (2.4) then allows to identify elements of the matrix algebra with functions on the classical space, and conversely the commutative space arise as a useful approximation of some matrix background. Its Riemannian structure will be identified later.

Note that given some arbitrary matrices $X^{a}$, there is in general no classical space for which this interpretation makes sense. Nevertheless, we will argue below that this class of backgrounds is in a sense stable and preferred by the matrix model action, and this concepts seems appropriate to understand the physical content of the matrix models under consideration here.

Let us discuss the semi-classical limit of a noncommutative space. In practical terms, this means that every matrix $F$ will be replaced by its classical pre-image $\mathscr{I}^{-1}(F)=: f$, and commutators will be replaced by Poisson brackets. The semi-classical limit provides the leading classical approximation of the noncommutative geometry, and will be denoted as $F \sim f$. However one can go beyond this semi-classical limit using e.g. the star product, which allows to systematically interpret the NC structure in the language of classical functions and geometry, as higher-order corrections in $\theta$ to the semi-classical limit. The matrix model action (1.1) can then be considered as a deformed action on some underlying classical space. This approximation is useful if the higherorder corrections in $\theta$ are small.

\section{Examples of matrix geometries}

In this section we discuss some basic examples of embedded noncommutative spaces described by finite or infinite matrix algebras. The salient feature is that the geometry is defined by a specific set of matrices $X^{a}$, interpreted as quantized embedding maps of a sub-manifold in $\mathbb{R}^{D}$.

\subsection{Prototype: the fuzzy sphere}

The fuzzy sphere $S_{N}^{2}[12,13]$ is a quantization or matrix approximation of the usual sphere $S^{2}$, with a cutoff in the angular momentum. We first note that the algebra of functions on the ordinary sphere can be generated by the coordinate functions $x^{a}$ of $\mathbb{R}^{3}$ modulo the relation $\sum_{a=1}^{3} x^{a} x^{a}=1$. The fuzzy sphere $S_{N}^{2}$ is a non-commutative space defined in terms of three $N \times N$ hermitian matrices $X^{a}, a=1,2,3$ subject to the relations

$$
\left[X^{a}, X^{b}\right]=\frac{i}{\sqrt{C_{N}}} \varepsilon^{a b c} X^{c}, \quad \sum_{a=1}^{3} X^{a} X^{a}=\mathbf{1}
$$


where $C_{N}=\frac{1}{4}\left(N^{2}-1\right)$ is the value of the quadratic Casimir of $\mathfrak{s u}(2)$ on $\mathbb{C}^{N}$. They are realized by the generators of the $N$-dimensional representation $(N)$ of $\mathfrak{s u}(2)$. The matrices $X^{a}$ should be interpreted as quantized embedding functions in the Euclidean space $\mathbb{R}^{3}$,

$$
X^{a} \sim x^{a}: \quad S^{2} \hookrightarrow \mathbb{R}^{3} .
$$

They generate an algebra $\mathscr{A} \cong \operatorname{Mat}(\mathrm{N}, \mathbb{C})$, which should be viewed as quantized algebra of functions on the symplectic space $\left(S^{2}, \omega_{N}\right)$ where $\omega_{N}$ is the canonical $S U(2)$-invariant symplectic form on $S^{2}$ with $\int \omega_{N}=2 \pi N$. The best way to see this is to decompose $\mathscr{A}$ into irreducible representations under the adjoint action of $S U(2)$, which is obtained from

$$
\begin{aligned}
S_{N}^{2} \cong(N) \otimes(\bar{N}) & =(1) \oplus(3) \oplus \ldots \oplus(2 N-1) \\
& =\left\{\hat{Y}_{0}^{0}\right\} \oplus \ldots \oplus\left\{\hat{Y}_{m}^{N-1}\right\} .
\end{aligned}
$$

This provides the definition of the fuzzy spherical harmonics $\hat{Y}_{m}^{l}$, and defines the quantization map

$$
\begin{aligned}
\mathscr{I}: \quad \mathscr{C}\left(S^{2}\right) & \rightarrow \mathscr{A}=\operatorname{Mat}(\mathrm{N}, \mathbb{C}) \\
Y_{m}^{l} & \mapsto\left\{\begin{array}{cc}
\hat{Y}_{m}^{l}, & l<N \\
0, & l \geq N
\end{array}\right.
\end{aligned}
$$

It follows easily that $\mathscr{I}\left(i\left\{x^{a}, x^{b}\right\}\right)=\left[X^{a}, X^{b}\right]$ where $\{$,$\} denotes the Poisson brackets correspond-$ ing to the symplectic form $\omega_{N}=\frac{N}{2} \varepsilon_{a b c} x^{a} d x^{b} d x^{c}$ on $S^{2}$. Together with the fact that $\mathscr{I}(f g) \rightarrow$ $\mathscr{I}(f) \mathscr{I}(g)$ for $N \rightarrow \infty$ (which is not hard to prove), $\mathscr{I}(i\{f, g\}) \stackrel{N \rightarrow \infty}{\rightarrow}[\mathscr{I}(f), \mathscr{I}(g)]$ follows. This means that $S_{N}^{2}$ is the quantization of $\left(S^{2}, \omega_{N}\right)$. It is also easy to see the following integral relation

$$
2 \pi \operatorname{Tr}(\mathscr{I}(f))=\int_{S^{2}} \omega_{N} f,
$$

consistent with (2.7). Therefore $S_{N}^{2}$ is the quantization of $\left(S^{2}, \omega_{N}\right)$. Moreover, there is a natural Laplace operator ${ }^{3}$ on $S_{N}^{2}$ defined as

$$
\square=\left[X^{a},\left[X^{b}, .\right]\right] \delta_{a b}
$$

which is invariant under $S U(2)$; in fact it is nothing but the quadratic Casimir of the $S U(2)$ action on $S^{2}$. It is then easy to see that up to normalization, its spectrum coincides with the spectrum of the classical Laplace operator on $S^{2}$ up to the cutoff, and the eigenvectors are given by the fuzzy spherical harmonics $\hat{Y}_{m}^{l}$.

In this special example, (3.3) allows to construct a series of embeddings of vector spaces

$$
\mathscr{A}_{N} \subset \mathscr{A}_{N+1} \subset \ldots
$$

with norm-preserving embedding maps. This allows to recover the classical sphere by taking the inductive limit. While this is a very nice structure, we do not want to rely on the existence of such explicit series of embeddings. We emphasize that even finite-dimensional matrices allow to approximate a classical geometry to a high precision, as discussed further in section 3.7.

\footnotetext{
${ }^{3}$ The symbol $\square$ is used here to distinguish the matrix Laplace operator from the Laplacian $\Delta$ on some Riemannian manifold. It does not indicate any particular signature.
} 


\subsection{The fuzzy torus}

The fuzzy torus $T_{N}^{2}$ can be defined in terms of clock and shift operators $U, V$ acting on $\mathbb{C}^{N}$ with relations $U V=q V U$ for $q^{N}=1$, with $U^{N}=V^{N}=1$. They have the following standard representation

$$
U=\left(\begin{array}{ccccc}
0 & 1 & 0 & \ldots & 0 \\
0 & 0 & 1 & \ldots & 0 \\
& \ddots & & \\
0 & \ldots & 0 & 1 \\
1 & 0 & \ldots & & 0
\end{array}\right), \quad V=\left(\begin{array}{cccc}
1 & & & \\
& e^{2 \pi i \frac{1}{N}} & & \\
& e^{2 \pi i \frac{2}{N}} & \\
& & \ddots & \\
& & & e^{2 \pi i \frac{N-1}{N}}
\end{array}\right)
$$

These matrices generate the algebra $\mathscr{A} \cong \operatorname{Mat}(\mathrm{N}, \mathbb{C})$, which can be viewed as quantization of the function algebra $\mathscr{C}\left(T^{2}\right)$ on the symplectic space $\left(T^{2}, \omega_{N}\right)$. One way to recognize the structure of a torus is by identifying a $\mathbb{Z}_{N} \times \mathbb{Z}_{N}$ symmetry, defined as

$$
\begin{aligned}
\mathbb{Z}_{N} \times \mathscr{A} & \rightarrow \mathscr{A} \\
\left(\omega^{k}, \phi\right) & \mapsto U^{k} \phi U^{-k}
\end{aligned}
$$

and similarly for the other $\mathbb{Z}_{N}$ defined by conjugation with $V$. Under this action, the algebra of functions $\mathscr{A}=\operatorname{Mat}(\mathrm{N}, \mathbb{C})$ decomposes as

$$
\mathscr{A}=\oplus_{n, m=0}^{N-1} U^{n} V^{m}
$$

into harmonics i.e. irreducible representations. This suggests to define the following quantization map:

$$
\begin{aligned}
\mathscr{I}: \quad \mathscr{C}\left(T^{2}\right) & \rightarrow \mathscr{A}=\operatorname{Mat}(\mathrm{N}, \mathbb{C}) \\
e^{i n \varphi} e^{i m \psi} & \mapsto\left\{\begin{array}{c}
q^{-n m / 2} U^{n} V^{m}, \quad|n|,|m|<N / 2 \\
0, \quad \text { otherwise }
\end{array}\right.
\end{aligned}
$$

which is compatible with the $\mathbb{Z}_{N} \times \mathbb{Z}_{N}$ symmetry and satisfies $\mathscr{I}\left(f^{*}\right)=\mathscr{I}(f)^{\dagger}$. The underlying Poisson structure on $T^{2}$ is given by $\left\{e^{i \varphi}, e^{i \psi}\right\}=\frac{2 \pi}{N} e^{i \varphi} e^{i \psi}$ (or equivalently $\{\varphi, \psi\}=-\frac{2 \pi}{N}$ ), and it is easy to verify the following integral relation

$$
2 \pi \operatorname{Tr}(\mathscr{I}(f))=\int_{T^{2}} \omega_{N} f, \quad \omega_{N}=\frac{N}{2 \pi} d \varphi d \psi
$$

consistent with (2.7). Therefore $T_{N}^{2}$ is the quantization of $\left(T^{2}, \omega_{N}\right)$.

The metric is an additional structure which goes beyond the mere concept of quantization. Here we obtain it by considering $T^{2}$ as embedded noncommutative space in $\mathbb{R}^{4}$, by defining 4 hermitian matrices

$$
X^{1}+i X^{2}:=U, \quad X^{3}+i X^{4}:=V
$$

which satisfy the relations

$$
\begin{aligned}
\left(X^{1}\right)^{2}+\left(X^{2}\right)^{2} & =1=\left(X^{3}\right)^{2}+\left(X^{4}\right)^{2}, \\
\left(X^{1}+i X^{2}\right)\left(X^{3}+i X^{4}\right) & =q\left(X^{3}+i X^{4}\right)\left(X^{1}+i X^{2}\right) .
\end{aligned}
$$


They can again be viewed as embedding maps

$$
X^{a} \sim x^{a}: \quad T^{2} \hookrightarrow \mathbb{R}^{4}
$$

and we can write $x^{1}+i x^{2}=e^{i \varphi}, x^{3}+i x^{4}=e^{i \psi}$ in the semi-classical limit. This allows to consider the matrix Laplace operator (3.6), and to compute its spectrum:

$$
\begin{aligned}
\square \phi & =\left[X^{a},\left[X^{b}, \phi\right]\right] \delta_{a b} \\
& =\left[U,\left[U^{\dagger}, \phi\right]\right]+\left[V,\left[V^{\dagger}, \phi\right]\right]=4 \phi-U \phi U^{\dagger}-U^{\dagger} \phi U-V \phi V^{\dagger}-V^{\dagger} \phi V \\
\square\left(U^{n} V^{m}\right) & =-c\left([n]_{q}^{2}+[m]_{q}^{2}\right) U^{n} V^{m} \sim-c\left(n^{2}+m^{2}\right) U^{n} V^{m}, \\
c & =\left(q^{1 / 2}-q^{-1 / 2}\right)^{2} \sim \frac{1}{N^{2}}
\end{aligned}
$$

where

$$
[n]_{q}=\frac{q^{n / 2}-q^{-n / 2}}{q^{1 / 2}-q^{-1 / 2}}=\frac{\sin (n \pi / N)}{\sin (\pi / N)} \sim n \quad \text { ("q-number") }
$$

Thus the spectrum of the matrix Laplacian (3.6) approximately coincides ${ }^{4}$ with the classical case below the cutoff. Therefore $T_{N}^{2}$ with the embedding defined via the above embedding (3.14) has indeed the geometry of a torus.

\subsection{Fuzzy $\mathbb{C} P^{N}$}

A straightforward generalization of the fuzzy sphere leads to the fuzzy complex projective space $\mathbb{C} P_{N}^{n}$, which is defined in terms of hermitian matrices $X^{a}, a=1,2, \ldots, n^{2}+n$ subject to the relations

$$
\left[X^{a}, X^{b}\right]=\frac{i}{\sqrt{C_{N}^{\prime}}} f_{c}^{a b} X^{c}, \quad d_{a b}^{c} X^{a} X^{b}=D_{N} X^{c}, \quad X_{a} X^{a}=\mathbf{1}
$$

(adopting a sum convention). Here $f_{c}^{a b}$ are the structure constants of $\mathfrak{s u}(n+1), d^{a b c}$ is the totally symmetric invariant tensor, and $C_{N}^{\prime}, D_{N}$ are group-theoretical constants which are not needed here. These relations are realized by the generators of $\mathfrak{s u}(n+1)$ acting on irreducible representations with highest weight $(N, 0, \ldots, 0)$ or $(0,0, \ldots, N)$, with dimension $d_{N}$. Again, the matrices $X^{a}$ should be interpreted as quantized embedding functions in the Euclidean space $\mathfrak{s u}(n+1) \cong \mathbb{R}^{n^{2}+n}$,

$$
X^{a} \sim x^{a}: \quad \mathbb{C} P^{n} \hookrightarrow \mathbb{R}^{n^{2}+n} .
$$

They generate an algebra $\mathscr{A} \cong \operatorname{Mat}\left(\mathrm{d}_{\mathrm{N}}, \mathbb{C}\right)$, which should be viewed as quantized algebra of functions on the symplectic space $\left(\mathbb{C} P^{n}, N \omega\right)$ where $\omega$ is the canonical $S U(n)$-invariant symplectic form on $\mathbb{C} P^{n}$. It is easy to write down a quantization map analogous to (3.4),

$$
\mathscr{I}: \quad \mathscr{C}\left(\mathbb{C} P^{n}\right) \rightarrow \mathscr{A}
$$

using the decomposition of $\mathscr{A}$ into irreducible representations of $\mathfrak{s u}(n+1)$. Again, there is a natural Laplace operator on $\mathbb{C} P_{N}^{n}$ defined as in (3.6) whose spectrum coincides with the classical one up to the cutoff. A similar construction can be given for any coadjoint orbit of a compact Lie group.

\footnotetext{
${ }^{4}$ It is interesting to note that momentum space is compactified here, reflected in the periodicity of $[n]_{q}$.
} 


\subsection{The Moyal-Weyl quantum plane}

The Moyal-Weyl quantum plane $\mathbb{R}_{\theta}^{2 n}$ is defined in terms of $2 n$ (infinite-dimensional) hermitian matrices $X^{a} \in \mathscr{L}(\mathscr{H})$ subject to the relations

$$
\left[X^{\mu}, X^{v}\right]=i \theta^{\mu v} \mathbf{1}
$$

where $\theta^{\mu v}=-\theta^{v \mu} \in \mathbb{R}$. Here $\mathscr{H}$ is a separable Hilbert space. This generates the ( $n$-fold tensor product of the) Heisenberg algebra $\mathscr{A}$ (or some suitable refinement of it, ignoring operatortechnical subtleties here), which can be viewed as quantization of the algebra of functions on $\mathbb{R}^{2 n}$ using e.g. the Weyl quantization map

$$
\begin{aligned}
\mathscr{I}: \quad \mathscr{C}\left(\mathbb{R}^{2 n}\right) & \rightarrow \mathbb{R}_{\theta}^{2 n} \subset \operatorname{Mat}(\infty, \mathbb{C}) \\
e^{i k_{\mu} x^{\mu}} & \mapsto e^{i k_{\mu} X^{\mu}} .
\end{aligned}
$$

Since plane waves are irreducible representations of the translation group, this map is again defined as intertwiner of the symmetry group as in the previous examples. Of course, the matrices $X^{\mu}$ should be viewed as quantizations of the classical coordinate functions $X^{\mu} \sim x^{\mu}: \mathbb{R}^{2 n} \rightarrow \mathbb{R}^{2 n}$. Similar as in quantum mechanics, it is easy to see that the noncommutative plane waves satisfy the Weyl algebra

$$
e^{i k_{\mu} X^{\mu}} e^{i p_{\mu} X^{\mu}}=e^{\frac{i}{2} \theta^{\mu v} k_{\mu} p_{v}} e^{i\left(k_{\mu}+p_{\mu}\right) X^{\mu}}
$$

It is also easy to obtain an explicit form for the star product defined by the above quantization map: it is given by the famous Moyal-Weyl star product,

$$
(f \star g)(x)=f(x) e^{\frac{i}{2} \theta^{\mu v} \overleftarrow{\partial}_{\mu} \vec{\partial}_{v}} g(x)
$$

in obvious notation. This gives e.g.

$$
\begin{aligned}
x^{\mu} \star x^{v} & =x^{\mu} x^{v}+\frac{i}{2} \theta^{\mu v} \\
{\left[x^{\mu}, x^{v}\right]_{\star} } & =i \theta^{\mu v}
\end{aligned}
$$

In this example, we note that

$$
\left[X^{\mu}, .\right]=: i \theta^{\mu v} \partial_{v}
$$

provides a reasonable definition of partial derivatives in terms of inner derivations on $\mathscr{A}$, provided $\theta^{\mu v}$ is non-degenerate (which we will always assume). This is justified by the observation $\left[X^{\mu}, e^{i k_{v} X^{v}}\right]=-\theta^{\mu v} k_{v} e^{i k_{\mu} X^{\mu}}$ together with the identification $\mathscr{I}$. Therefore these partial derivatives and in particular the matrix Laplacian

$$
\begin{aligned}
\square & =\left[X^{\mu},\left[X^{v}, .\right]\right] \delta_{\mu v}=-\theta^{\mu \mu^{\prime}} \theta^{v v^{\prime}} \delta_{\mu v} \partial_{\mu^{\prime}} \partial_{v^{\prime}} \equiv-\Lambda_{\mathrm{NC}}^{-4} G^{\mu v} \partial_{\mu} \partial_{v}, \\
G^{\mu v} & :=\Lambda_{\mathrm{NC}}^{4} \theta^{\mu \mu^{\prime}} \theta^{v v^{\prime}} \delta_{\mu^{\prime} v^{\prime}}, \quad \Lambda_{\mathrm{NC}}^{4}:=\sqrt{\operatorname{det} \theta_{\mu \nu}^{-1}}
\end{aligned}
$$

coincide via $\mathscr{I}$ with the commutative Laplacian for the metric $G_{\mu \nu}$. Therefore $G_{\mu \nu}$ should be considered as effective metric of $\mathbb{R}_{\theta}^{4}$.

The Moyal-Weyl quantum plane differs from our previous examples in one essential way: the underlying classical space is non-compact. This means that the matrices become unbounded 
operators acting on an infinite-dimensional separable Hilbert space. The basic difference can be seen from the formula

$$
(2 \pi)^{n} \operatorname{Tr} F \sim \int_{\mathscr{M}}\left|\theta_{\mu \nu}^{-1}\right| f,
$$

which together with $\left|\theta_{\mu \nu}^{-1}\right|=$ const implies that the trace diverges as a consequence of the infinite symplectic volume. Locally (i.e. for test-functions $f$ with compact support, say), there is no essential difference between the compact fuzzy spaces described before and the Moyal-Weyl quantum plane. This reflects the Darboux theorem, which states that all symplectic spaces of a given dimension are locally equivalent. Thus from the point of view of matrix geometry, $\mathbb{R}_{\theta}^{2 n}$ is simply a non-compact version of a fuzzy space.

\subsection{The fuzzy cylinder}

Finally, the fuzzy cylinder $S^{1} \times_{\xi} \mathbb{R}$ is defined by [14]

$$
\begin{aligned}
{\left[X^{1}, X^{3}\right] } & =i \xi X^{2}, & {\left[X^{2}, X^{3}\right] } & =-i \xi X^{1}, \\
\left(X^{1}\right)^{2}+\left(X^{2}\right)^{2} & =R^{2}, & {\left[X^{1}, X^{2}\right] } & =0 .
\end{aligned}
$$

Defining $U:=X^{1}+i X^{2}$ and $U^{\dagger}:=X^{1}-i X^{2}$, this can be stated more transparently as

$$
\begin{aligned}
U U^{\dagger} & =U^{\dagger} U \quad=R^{2} \\
{\left[U, X^{3}\right] } & =\xi U, \quad\left[U^{\dagger}, X^{3}\right]=-\xi U^{\dagger}
\end{aligned}
$$

This algebra has the following irreducible representation ${ }^{5}$

$$
\begin{array}{rlrl}
U|n\rangle & =R|n+1\rangle, & U^{\dagger}|n\rangle & =R|n-1\rangle \\
X^{3}|n\rangle & =\xi n|n\rangle, & n \in \mathbb{Z}, \xi \in \mathbb{R}
\end{array}
$$

on a Hilbert space $\mathscr{H}$, where $|n\rangle$ form an orthonormal basis. We take $\xi \in \mathbb{R}$, since the $X^{i}$ are hermitian. Then the matrices $\left\{X^{1}, X^{2}, X^{3}\right\}$ can be interpreted geometrically as quantized embedding functions

$$
\left(\begin{array}{c}
X^{1}+i X^{2} \\
X^{3}
\end{array}\right) \sim\left(\begin{array}{c}
R e^{i y_{3}} \\
x^{3}
\end{array}\right): \quad S^{1} \times \mathbb{R} \hookrightarrow \mathbb{R}^{3} .
$$

The quantization map is given by

$$
\begin{aligned}
\mathscr{I}: \quad \mathscr{C}\left(S^{1} \times \mathbb{R}\right) & \rightarrow S^{1} \times \xi \mathbb{R} \subset \operatorname{Mat}(\infty, \mathbb{C}) \\
e^{i p x^{3}} e^{i n y^{3}} & \mapsto e^{i n \xi / 2} e^{i p X^{3}} U^{n},
\end{aligned}
$$

which preserves the obvious $U(1) \times \mathbb{R}$ symmetry. This defines the fuzzy cylinder $S^{1} \times \xi \mathbb{R}$. It is the quantization of $T^{*} S^{1}$ with canonical Poisson bracket $\left\{e^{i y_{3}}, x^{3}\right\}=-i \xi e^{i y_{3}}$, or $\left\{x^{3}, y^{3}\right\}=\xi$ locally. Its geometry can be recognized either using the $U(1) \times \mathbb{R}$ symmetry, or using the matrix Laplacian $\square=\left[X^{a},\left[X^{b},.\right]\right] \delta_{a b}$ which has the following spectrum

$$
\square e^{i p X^{3}} U^{n}=\left(4 R^{2} \sin ^{2}(p \xi / 2)+n^{2} \xi^{2}\right) e^{i p X^{3}} U^{n} \stackrel{p \xi \ll 1}{\sim}\left(R^{2} p^{2}+n^{2}\right) \xi^{2} e^{i p X^{3}} U^{n},
$$

consistent with the classical spectrum for small momenta. Therefore the effective geometry is that of a cylinder.

\footnotetext{
${ }^{5}$ More general irreducible representations are obtained by a (trivial) constant shift $X^{3} \rightarrow X^{3}+c$.
} 


\subsection{Additional structures: coherent states and differential calculus}

Next we exhibit some additional results in the example of the fuzzy sphere. They can be generalized to other matrix geometries under consideration here.

Coherent states. As in Quantum Mechanics, coherent states provide a particularly illuminating way to understand quantum geometry, via maximally localized wave-functions. In the case of $S_{N}^{2}$ they go back to Perelomov [15], although different approaches are possible [16]. Here we consider the group-theoretical approach of Perelomov. Let $p_{0} \in S^{2}$ be a given point on $S^{2}$ called north pole. Then the map

$$
\begin{aligned}
S O(3) & \rightarrow S^{2} \\
g & \mapsto g \triangleright p_{0}
\end{aligned}
$$

defines the stabilizer group $U(1) \subset S O(3)$ of $p_{0}$, so that $S^{2} \cong S U(2) / U(1)$. Now recall that the algebra of functions on the fuzzy sphere is given by $\operatorname{End}(\mathscr{H})$, where $\mathscr{H}$ is spanned by the angular momentum basis $|m, L\rangle, m=-L, \ldots, L, N=2 L+1$. Noting that $X^{3}|L, L\rangle=\frac{N-2}{\sqrt{N^{2}-1}}|L, L\rangle$, we can identify the highest weight state $|L, L\rangle$ as coherent state localized on the north pole. We define more generally

$$
\left|\psi_{g}\right\rangle:=\pi_{N}(g)|L, L\rangle
$$

where $\pi_{N}$ denotes the $N$-dimensional irrep of $S O(3)$. Since the stabilizer group $U(1)$ of $p_{0}$ acts on $\left|\psi_{g}\right\rangle$ via a complex phase, the associated one-dimensional projector

$$
\Pi_{g}=\left|\psi_{g}\right\rangle\left\langle\psi_{g}\right|
$$

is independent of $U(1)$, so that there is a well-defined map

$$
\begin{aligned}
S O(3) / U(1) \cong S^{2} & \rightarrow \operatorname{End}(\mathscr{H}) \\
p & \mapsto \Pi_{g}=\left|\psi_{g}\right\rangle\left\langle\psi_{g}\right|=:(4 \pi)^{-1} \delta_{N}^{(2)}(p-x) .
\end{aligned}
$$

The notation on the rhs should indicate that these are the optimally localized wave-functions on $S_{N}^{2}$. To proceed, we label coherent states related by $U(1)$ with the corresponding point on $S^{2}$, so that $|p\rangle:=\left|\psi_{g(p)}\right\rangle \sim\left|\psi_{g(p)^{\prime}}\right\rangle$. One can then show the following results [15]

$$
\begin{aligned}
& \int_{S^{2}}|p\rangle\langle p|=c 1 \quad \text { overcomplete } \\
& \left|\left\langle p \mid p^{\prime}\right\rangle\right|=(\cos (\vartheta / 2))^{N-1}, \quad \vartheta=\measuredangle\left(p, p^{\prime}\right) \quad \text { localization } p \approx p^{\prime} \\
& p_{a} X^{a}|p\rangle=|p\rangle \\
& \left\langle p\left|X^{a}\right| p\right\rangle=\operatorname{Tr} X^{a} \Pi_{p}=p^{a} \in S^{2}
\end{aligned}
$$

which provide a justification for the interpretation of $X^{a} \sim x^{a}: S^{2} \hookrightarrow \mathbb{R}^{3}$ (3.2) as quantized embedding maps. One can also show that these states are optimally localized on $S^{2}$,

$$
\left(\Delta X^{1}\right)^{2}+\left(\Delta X^{2}\right)^{2}+\left(\Delta X^{3}\right)^{2} \geq \frac{N-1}{2 C_{N}} \sim \frac{1}{2 N}=\sum_{a}\left\langle p\left|\left(X^{a}-\left\langle p\left|X^{a}\right| p\right\rangle\right)^{2}\right| p\right\rangle .
$$

In principle, such considerations should apply to all matrix geometries under consideration here, although the explicit realization of such coherent states is in general not known. 
Differential calculus. For many noncommutative spaces with enhanced symmetry, differential calculi have been constructed which respect the symmetry. In the case of embedded NC spaces as considered here, this calculus is typically that of the embedding space $\mathbb{R}^{D}$, and does not reduce to the standard one in the commutative limit. Nevertheless it may be quite appealing, and we briefly discuss the example of the fuzzy sphere following Madore [12].

A differential calculus on $S_{N}^{2}$ is a graded bimodule $\Omega_{N}^{\star}=\oplus_{n \geq 0} \Omega^{n}$ over $\Omega^{0}=\mathscr{A}=S_{N}^{2}$ with an exterior derivative $d: \Omega^{n} \rightarrow \Omega^{n+1}$ compatible with $S O(3)$, which satisfies $d^{2}=0$ and the graded Leibniz rule $d(\alpha \beta)=d \alpha \beta+(-1)^{|\alpha|} \alpha d \beta$. Since $\left[X^{a}, X^{b}\right] \sim i \varepsilon^{a b c} X^{c}$ one necessarily has $d X^{a} X^{b} \neq$ $X^{b} d X^{a}$ and $d X^{a} d X^{b} \neq-d X^{b} d X^{a}$. It turns out that there is a preferred (radial) one-form which generates the exterior derivative ${ }^{6}$ on $\mathscr{A}$,

$$
d f=[\omega, f], \quad \omega=-C_{N} X^{a} d X^{a} .
$$

It turns out that the calculus necessarily contains 3 -forms

$$
\Omega_{N}^{\star}=\oplus_{n=0}^{3} \Omega_{N}^{n}, \quad \Omega_{N}^{3} \ni f_{a b c}(X) d X^{a} d X^{b} d X^{c},
$$

which reflects the embedding space $\mathbb{R}^{3}$. It turns out that can introduce a frame of one-forms which commute with all functions [12]:

$$
\xi^{a}=\omega X^{a}+\sqrt{C_{N}} \varepsilon^{a b c} X^{b} d X^{c}, \quad\left[f(X), \xi^{a}\right]=0 .
$$

The most general one-form can then be written as

$$
A=A_{a} \xi^{a} \quad \in \Omega_{N}^{1}, \quad A_{a} \in \mathscr{A}=\operatorname{Mat}(\mathrm{N}, \mathbb{C}) .
$$

One can define the exterior derivative such that [17]

$$
\begin{aligned}
& F=d A+A A=\left(Y_{a} Y_{b}+i \varepsilon_{a b c} Y_{c}\right) \xi^{a} \xi^{b} \quad \in \Omega_{N}^{2} \\
& Y=\omega+A=Y_{a} \xi^{a}=\left(X_{a}+A_{a}\right) \xi^{a} \in \Omega_{N}^{1} .
\end{aligned}
$$

These formulae are relevant in the context of gauge theory, encoding the covariant coordinates $Y^{a}=X^{a}+A^{a}$ which are the basic objects in matrix models. Nevertheless the formalism of differential forms may be somewhat misleading, because the one-form $Y$ encodes both tangential gauge fields as well as transversal scalar fields. In any case we will not use differential calculi in the following. Our aim is to understand the geometrical structures which emerge from matrix models, without introducing any mathematical prejudice. These models do not require any such additional mathematical structures.

This concludes our brief exhibition of matrix geometries, through examples whose geometry was identified using their symmetry properties. We will learn below how to generalize them for generic geometries, and how to systematically extract their geometry without using this symmetry. In particular, the form of the matrix Laplacian (3.6) turns out to be general. On the other hand, there are also more exotic and singular spaces that can be modeled by matrices, such as intersecting spaces, stacks of spaces, etc. Some well-known NC spaces such as $\kappa$-Minkowski space are

\footnotetext{
${ }^{6}$ This formula is modified for higher forms, cf. [17]. $\omega$ should not be confused with the symplectic form.
} 
quantizations of degenerate Poisson structures, which we do not consider since the effective metric would be degenerate and/or singular. There are also very different types of noncommutative tori [18] described by infinite-dimensional algebras, reflecting the presence of a non-classical winding sector. They are not stable under small deformations, e.g. it is crucial whether $\theta$ is rational or irrational. In contrast, the embedded NC spaces considered here such as fuzzy tori are stable under deformations as explained in section 7 , and contain no winding modes. This seems crucial for supporting a well-defined quantum field theory, as discussed in section 10 .

Finally we should recall the noncommutative geometry as introduced by A. Connes [9], which is based on a Dirac operator $\not D$ subject to certain axioms. One can define a differential calculus based on such a Dirac operator, which is a refined version of $d \sim[\not D,$.$] . The matrix models$ discussed below indeed provide a Dirac operator for the matrix geometries under consideration, although these axioms are not necessarily respected.

\subsection{Lessons and cautions}

We draw the following general lessons from the above examples:

- The algebra $\mathscr{A}=\mathscr{L}(\mathscr{H})$ of linear operators on $\mathscr{H}$ should be viewed as quantization of the algebra of functions on a symplectic space $(\mathscr{M}, \omega)$. However as abstract algebra, $\mathscr{A}$ carries no geometrical information, not even the dimension or the topology of the underlying space.

- The geometrical information is encoded in the specific matrices $X^{a}$, which should be interpreted as quantized embedding functions

$$
X^{a} \sim x^{a}: \quad \mathscr{M} \hookrightarrow \mathbb{R}^{D} .
$$

They encode the embedding geometry, which is contained e.g. in the matrix Laplacian. We will learn below how to extract this more directly. The Poisson or symplectic structure is encoded in their commutation relations. In this way, even finite-dimensional matrices can describe various geometries to a high precision.

- In some sense, every non-degenerate and "regular" fuzzy space given by the quantization of a symplectic manifold locally looks like some $\mathbb{R}_{\theta}^{2 n}$. The algebra of functions on $\mathbb{R}_{\theta}^{2 n}$ is infinitedimensional only because its volume is infinite: $\operatorname{dim}(\mathscr{H})$ counts the number of quantum cells via the Bohr-Sommerfeld quantization rule, which is nothing but the semi-classical relation (2.7). For example, $\mathbb{C} P_{N}^{n}$ can be viewed as a compactification of $\mathbb{R}_{\theta}^{2 n}$.

This leads to the idea that generic geometries may be described similarly as embedded noncommutative spaces in matrix models, interpreting the matrices $X^{a}$ as quantized embedding maps $X^{a} \sim x^{a}: \mathscr{M} \hookrightarrow \mathbb{R}^{D}$. However, we caution that general matrices do not necessarily admit a geometrical interpretation. There is not even a notion of dimension in general. In fact we will see that matrix models can describe much more general situations, such as multiple submanifolds ("branes"), intersecting branes, manifolds suspended between branes, etc., essentially the whole zoo of string theory. Therefore we have to make some simplifying assumptions, and focus on the simplest case of NC branes corresponding to smooth submanifolds. This will be justified in section 7 by showing 
that such configurations are stable under small deformations. Moreover, we will explain in section 6 how to realize a large class of generic $4 \mathrm{~d}$ geometries through such matrix geometries.

A sharp separation between admissible and non-admissible matrix geometries would in fact be inappropriate in the context of matrix model, whose quantization is defined in terms of an integral over the space of all matrices as discussed below. The ultimate aim is to show that the dominant contributions to this integral correspond to matrix configurations which have a geometrical meaning relevant to physics. However, the integral is over all possible matrices, including geometries with different dimensions and topologies. It is therefore clear that such a geometric notion can only be approximate or emergent.

Finally, we wish to address the issue of finite-dimensional versus infinite-dimensional matrix algebras. Imagine that our space-time was fuzzy with a UV scale $\Lambda_{\mathrm{NC}} \approx \Lambda_{\text {Planck }}$, and compact of size $R$. Then there would be only finitely many "quantum cells", and the geometry should be modeled by some finite $N$-dimensional (matrix) algebra. Since no experiment on earth can directly access the Planck scale, such a scenario can hardly be ruled out, and a model based on a finite matrix geometry might be perfectly adequate. Therefore the limit $N \rightarrow \infty$ or $\Lambda_{\mathrm{NC}} \rightarrow \infty$ may not be realized in physics. However there must be a large "separation of scales", and this limits should be well-behaved in order to have any predictability; whether or not the limit is realized in nature is then irrelevant.

\section{Spectral matrix geometry}

We want to understand more generally such matrix geometries, described by a number of hermitian matrices $X^{a} \in \mathscr{A}=\mathscr{L}(\mathscr{H})$. Here $\mathscr{H}$ is a finite-dimensional or infinite-dimensional (separable) Hilbert space.

One way to extract geometrical information from a space $\mathscr{M}$ which naturally generalizes to the noncommutative setting is via spectral geometry. In the classical case, one can consider the heat kernel expansion of the Laplacian $\Delta_{g}$ of a compact Riemannian manifold $(\mathscr{M}, g)$ [19],

$$
\operatorname{Tr} e^{-\alpha \Delta_{g}}=\sum_{n \geq 0} \alpha^{(n-d) / 2} \int_{\mathscr{M}} d^{d} x \sqrt{|g|} a_{n}(x) .
$$

The Seeley-de Witt coefficients $a_{n}(x)$ of this asymptotic expansion are determined by the intrinsic geometry of $\mathscr{M}$, e.g. $a_{2} \sim-\frac{R[g]}{6}$ where $R[g]$ is the curvature scalar. This provides physically valuable information on $\mathscr{M}$, and describes the one-loop effective action. In particular, the leading term allows to compute the number of eigenvalues below some cutoff,

$$
\mathscr{N}_{\Delta}(\Lambda):=\#\left\{\mu^{2} \in \operatorname{spec} \Delta ; \mu^{2} \leq \Lambda^{2}\right\}
$$

dropping the subscript $g$ of the Laplacian. One obtains Weyls famous asymptotic formula

$$
\mathscr{N}_{\Delta}(\Lambda) \sim c_{d} \operatorname{vol} \mathscr{M} \Lambda^{d}, \quad c_{d}=\frac{\operatorname{vol} S^{d-1}}{d(2 \pi)^{d}} .
$$

In particular, the (spectral) dimension $d$ of $\mathscr{M}$ can be extracted the from the asymptotic density of the eigenvalues of $\Delta_{g}$. However, although the spectrum of $\Delta_{g}$ contains a lot of information on 
the geometry, it does not quite determine it uniquely, and there are inequivalent but isospectral manifolds ${ }^{7}$.

Now consider the spectral geometry of fuzzy spaces in more detail. Since the asymptotic density of eigenvalues vanishes in the compact case, the proper definition of a spectral dimension in the fuzzy case with Laplacian $\square$ must take into account the cutoff, e.g. as follows

$$
\mathscr{N}_{\square}(\Lambda) \sim c_{d} \operatorname{vol} \mathscr{M} \Lambda^{d} \quad \text { for } \Lambda \leq \Lambda_{\max }
$$

where $\Lambda_{\max }$ is the (sharp or approximate) cutoff of the spectrum. Similarly, the information about the geometry of $\mathscr{M}$ is encoded in the spectrum of its Laplacian or Dirac operator below its cutoff. It turns out that such a cutoff is in fact essential to obtain meaningful Seeley-de Witt coefficients in the noncommutative case [21]. We can thus formulate a specific way to associate an effective geometry to a noncommutative space with Laplacian $\square$ : if $\operatorname{spec} \square$ has a clear enough asymptotics for $\Lambda \leq \Lambda_{\max }$ and approximately coincides with $\operatorname{spec} \Delta_{g}$ for some classical manifold $(\mathscr{M}, g)$ for $\Lambda \leq \Lambda_{\max }$, then its spectral geometry is that of $(\mathscr{M}, g)$.

To proceed, we need to specify a Laplacian for matrix geometries. Here the (Yang-Mills) matrix model (1.1) provides a natural choice: For any given background configuration in the matrix model defined by $D$ hermitian matrices $X^{a}$, there is a natural matrix Laplace operator ${ }^{8}$

$$
\square=\left[X^{a},\left[X^{b}, .\right]\right] \delta_{a b}
$$

which is a (formally) hermitian operator on $\mathscr{A}$. We can study its spectrum and the distribution of eigenvalues. This Laplacian governs the fluctuations in the matrix model, and therefore encodes its effective geometry. Hence if there is a classical geometry which effectively describes the matrix background $X^{a}$ up to some scale $\Lambda_{\mathrm{NC}}$, the spectrum of its canonical (Levi-Civita) Laplacian $\Delta_{g}$ must approximately coincide with the spectrum of $\square$, up to some possible cutoff $\Lambda$. In particular, there should be a refined version of the quantization map (2.4)

$$
\begin{aligned}
\mathscr{I}: \quad \mathscr{C}_{\Lambda}(\mathscr{M}) & \rightarrow \mathscr{A} \subset \operatorname{Mat}(\infty, \mathbb{C}) \\
f(x) & \mapsto F
\end{aligned}
$$

which approximately intertwines the Laplacians $\mathscr{I}\left(\Delta_{g} f\right) \approx \square(\mathscr{I}(f))$. Here $\mathscr{C}_{\Lambda}(\mathscr{M})$ denotes the space of functions on $\mathscr{M}$ whose eigenvalues are bounded by $\Lambda$, and $\mathscr{I}$ should be injective. The fuzzy sphere is an example where the matrix Laplacian precisely matches the classical Laplacian up to the cutoff. Its special symmetry is no longer essential.

\section{Embedded noncommutative spaces and their geometry.}

In practice, it is hard to extract information on the metric from the spectrum. A more direct handle on the geometry can be obtained for embedded noncommutative spaces, which can be understood as quantization of an approximate classical symplectic manifold $\left(\mathscr{M}, \theta^{\mu v}\right)$ embedded in $\mathbb{R}^{D}$. This makes matrix models much more accessible than abstract NC geometry.

\footnotetext{
${ }^{7}$ One way to close this gap is to consider spectral triples associated to Dirac operators [20]. In the matrix model, the geometrical information will be extracted more directly using the symplectic structure and the embedding defined by the matrices $X^{a}$.

${ }^{8}$ This operator arises e.g. as equation of motion for the Yang-Mills matrix model. There is also a natural matrix Dirac operator $\not D \Psi=\Gamma_{a}\left[X^{a}, \Psi\right]$ where $\Gamma_{a}$ generates the Clifford algebra of $S O(D)$. However we will not discuss it here.
} 
Thus consider again matrices $X^{a}$ which can be viewed as quantized Cartesian embedding functions of $\mathscr{M}$ in $\mathbb{R}^{D}$,

$$
X^{a} \sim x^{a}: \mathscr{M} \hookrightarrow \mathbb{R}^{D} .
$$

One can then interpret commutators as quantization of the Poisson structure on $\mathscr{M}$. In particular,

$$
i \Theta^{a b}=\left[X^{a}, X^{b}\right] \sim i\left\{x^{a}, x^{b}\right\}=i \theta^{\mu v} \partial_{\mu} x^{a} \partial_{\nu} x^{b}
$$

in the semi-classical limit, where $\theta^{\mu v}$ is the Poisson tensor in some local coordinates on $\mathscr{M}$. It is then not hard to see [5] that

$$
\square \phi \equiv\left[X^{a},\left[X^{b}, \phi\right]\right] \delta_{a b} \sim-\left\{X^{a},\left\{X^{b}, \phi\right\}\right\} \delta_{a b}=-e^{\sigma} \Delta_{G} \phi(x)
$$

for any matrix resp. function $\mathscr{A} \ni \phi \sim \phi(x)$. Here $\Delta_{G}$ is the standard Laplace operator associated to the effective metric $G_{\mu \nu}$ defined as follows

$$
\begin{aligned}
G^{\mu v}(x) & :=e^{-\sigma} \theta^{\mu \mu^{\prime}}(x) \theta^{v v^{\prime}}(x) g_{\mu^{\prime} v^{\prime}}(x) \\
g_{\mu v}(x) & :=\partial_{\mu} x^{a} \partial_{\nu} x^{b} \delta_{a b}, \\
e^{-(n-1) \sigma} & :=\frac{1}{\theta^{n}}\left|g_{\mu v}(x)\right|^{-\frac{1}{2}}, \quad \theta^{n}=\left|\theta^{\mu v}\right|^{1 / 2} .
\end{aligned}
$$

All of these are tensorial objects on $\mathscr{M}$, e.g. $g_{\mu v}(x)$ is the metric induced on $\mathscr{M} \subset \mathbb{R}^{D}$ via pull-back of $\delta_{a b}$. The normalization factor $e^{-\sigma}$ is determined uniquely (except for $n=1$ ) such that

$$
\frac{1}{\theta^{n}}=\sqrt{\left|G_{\mu v}\right|} e^{-\sigma}
$$

This provides the desired explicit description of the matrix geometry at the semi-classical level. It is easy to check that $G_{\mu \nu}=g_{\mu \nu}$ for the examples in section 3, which will be understood on more general grounds below.

The easiest way to see (5.3) is by considering the action for a scalar field coupled to the matrix model background

$$
S[\varphi] \equiv-\operatorname{Tr}\left[X^{a}, \phi\right]\left[X^{b}, \phi\right] \delta_{a b} \sim \frac{1}{(2 \pi)^{n}} \int d^{2 n} x \sqrt{\left|G_{\mu v}\right|} G^{\mu v}(x) \partial_{\mu} \phi \partial_{\nu} \phi .
$$

Writing the lhs as $\operatorname{Tr} \phi \square \phi$ and taking the semi-classical limit leads to (5.3). Note that this is the action for additional matrix components $\phi \equiv X^{D+1}$ in the matrix model (1.1). Therefore $G_{\mu \nu}$ is the metric which governs scalar fields in the matrix model, more precisely nonabelian scalar fields which arise as transversal fluctuations on backgrounds $X^{a} \otimes \mathbf{1}_{n}$, cf. (8.2). More generally, one can show that all fields which arise in the matrix model as fluctuations of the matrices around such a background (i.e. scalar fields, gauge fields and fermions) are governed by $G_{\mu \nu}$, possibly up to a conformal factor $\sim e^{\sigma}$. This means that $G_{\mu \nu}$ is the effective gravitational metric.

Now consider the equations of motion of the matrix model (1.1), which are given by

$$
0=\left[X^{b},\left[X^{b^{\prime}}, X^{a}\right]\right] g_{b b^{\prime}}=\square X^{a} \sim-e^{\sigma} \Delta_{G} x^{a} .
$$

This means that the embedding functions are harmonic functions w.r.t. $G_{\mu \nu}$, which is satisfied e.g. for $\mathbb{R}_{\theta}^{2 n}$. With a little more effort, one can also derive the following equations for the Poisson structure $[29,5]$

$$
\nabla_{G}^{\mu}\left(e^{\sigma} \theta_{\mu v}^{-1}\right)=e^{-\sigma} G_{v \rho} \theta^{\rho \mu} \partial_{\mu} \eta, \quad \eta=\frac{1}{4} e^{\sigma} G^{\mu v} g_{\mu \nu}
$$


However, quantum corrections are expected to be essential for the gravity sector, and one should be careful avoiding preliminary conclusions. In any case, we note the following observations:

- Assume that $\operatorname{dim} \mathscr{M}=4$. Then $G_{\mu \nu}=g_{\mu \nu}$ if and only if the symplectic form $\omega=\frac{1}{2} \theta_{\mu \nu}^{-1} d x^{\mu} d x^{\nu}$ is self-dual or anti-selfdual [5].

- There is a natural tensor

$$
\mathscr{J}_{\gamma}^{\eta}=e^{-\sigma / 2} \theta^{\eta \gamma^{\prime}} g_{\gamma^{\prime} \gamma}=-e^{\sigma / 2} G^{\eta \gamma^{\prime}} \theta_{\gamma^{\prime} \gamma}^{-1}
$$

Then the effective metric can be written as

$$
G^{\mu v}=\mathscr{J}_{\rho}^{\mu} \mathscr{J}_{\rho^{\prime}}^{v} g^{\rho \rho^{\prime}}=-\left(\mathscr{J}^{2}\right)_{\rho}^{\mu} g^{\rho v} .
$$

In particular, $\mathscr{J}$ defines an almost-complex structure if and only if $G_{\mu v}=g_{\mu v}$, hence for (anti-)selfdual $\omega$. In that case, $(\mathscr{M}, \tilde{g}, \omega)$ defines an almost-Kähler structure on $\mathscr{M}$ where

$$
\tilde{g}_{\mu v}:=e^{-\sigma / 2} g_{\mu \nu} .
$$

- The matrix model is invariant under gauge transformations $X^{a} \rightarrow X^{a \prime}=U^{-1} X^{a} U$, which semi-classically correspond to symplectomorphisms $\Psi_{U}$ on $(\mathscr{M}, \omega)$. This can be viewed in terms of modified embeddings $x^{a \prime}=x^{a} \circ \Psi_{U}: \mathscr{M} \rightarrow \mathbb{R}^{D}$ with equivalent geometry.

- Matrix expressions such as $\left[X^{a}, X^{b}\right] \sim i \theta^{\mu v} \partial_{\mu} x^{a} \partial_{v} x^{b}$ should be viewed as (quantizations of) tensorial objects on $\mathscr{M} \subset \mathbb{R}^{D}$, written in terms of Cartesian coordinates $a, b$ of the ambient space $\mathbb{R}^{D}$. They are always tangential because $\partial_{\nu} x^{b} \in T_{p} \mathscr{M}$. Using appropriate projectors on the tangential and normal bundles of $\mathscr{M}$, this can be used to derive matrix expressions which encode e.g. the intrinsic curvature of $\mathscr{M}$, cf. [22, 23]. This is important for gravity.

\section{Realization of generic $4 \mathrm{D}$ geometries in matrix models}

We now show how a large class of generic (Euclidean, for now) 4-dimensional geometries can be realized as NC branes in matrix models with $D=10$. This should eliminate any lingering doubts whether the geometries in the matrix model are sufficiently general for gravity. This construction is illustrated in [24] for the example of the Schwarzschild geometry.

1. Consider some "reasonable" generic geometry $\left(\mathscr{M}^{4}, g_{\mu \nu}\right)$ with nice properties, as explained below.

2. Choose an embedding $\mathscr{M} \hookrightarrow \mathbb{R}^{D}$. This is in general not unique, and requires that $D$ is sufficiently large. Using classical embedding theorems [25], $D=10$ is just enough to embed generic 4-dimensional geometries (at least locally).

3. Equip $\mathscr{M}$ with an (anti-)selfdual closed 2-form $\omega$. This means that $d \omega=d \star_{g} \omega=0$, hence $\omega$ is a special solution of the free Maxwell equations on $\mathscr{M}$. Such a solution generically exists for mild assumptions on $\mathscr{M}$, for example by solving the corresponding boundary value 
problem with $\omega$ being (anti-)selfdual on the boundary or asymptotically ${ }^{9}$. This defines the requirements in step 1). For asymptotically flat spaces, $\omega$ should be asymptotically constant in order to ensure that $e^{-\sigma}$ is asymptotically constant. This requirement can be met more easily in the presence of compact extra dimensions $\mathscr{M}^{4} \times \mathscr{K}$ [26].

As explained above, it follows that $(\tilde{g}, \omega)(5.13)$ is almost-Kähler. Under mild assumptions, one can then show [27] that there exists a quantization (2.4) of the symplectic space $(\mathscr{M}, \omega)$ in terms of operators on a Hilbert space ${ }^{10}$. In particular, we can then define $X^{a}:=\mathscr{I}\left(x^{a}\right) \in \mathscr{A}$ to be the matrix obtained as quantization of $x^{a}$, so that

$$
X^{a} \sim x^{a}: \mathscr{M} \rightarrow \mathbb{R}^{D} .
$$

The effective metric on $\mathscr{M}$ is therefore $G_{\mu \nu}$ as explained above.

4. Since $\omega$ is (anti-)selfdual it follows that $G=g$, and we have indeed obtained a quantization of $(\mathscr{M}, g)$ in terms of a matrix geometry. In particular, the matrix Laplacian $\square$ will approximate $\Delta_{g}$ for low enough eigenvalues, and fluctuations of the matrix model around this background describe fields propagating on this effective geometry.

\section{Deformations of embedded NC spaces}

Assume that $X^{a} \sim x^{a}: \mathscr{M} \hookrightarrow \mathbb{R}^{D}$ describes some quantized embedded space as before. The important point which justifies the significance of this class of configurations is that it is preserved by small deformations. Indeed, consider a small deformation $\tilde{X}^{a}=X^{a}+A^{a}$ by generic matrices $A^{a} \in \mathscr{A}$. By assumption, there is a local neighborhood for any point $p \in \mathscr{M}$ where we can separate the matrices $X^{a}$ into independent coordinates and embedding functions,

$$
X^{a}=\left(X^{\mu}, \phi^{i}\left(X^{\mu}\right)\right)
$$

such that the $X^{\mu}$ generate the full ${ }^{11}$ matrix algebra $\mathscr{A}$. Therefore we can write $A^{a}=A^{a}\left(X^{\mu}\right)$, and assume that it is smooth (otherwise the deformation will be suppressed by the action). We can now consider $\tilde{X}^{\mu}=X^{\mu}+A^{\mu} \sim \tilde{x}^{\mu}\left(x^{v}\right)$ as new coordinates with modified Poisson structure $\left[\tilde{X}^{\mu}, \tilde{X}^{v}\right] \sim i\left\{\tilde{x}^{\mu}, \tilde{x}^{v}\right\}$, and $\tilde{\phi}^{i}=\phi^{i}+A^{i} \sim \tilde{\phi}^{i}\left(\tilde{x}^{\mu}\right)$ as modified embedding of $\tilde{\mathscr{M}} \hookrightarrow \mathbb{R}^{D}$. Therefore $\tilde{X}^{a}$ describes again a quantized embedded space. Due to this stability property, it is plausible that the class of embedded NC spaces plays a dominant role in the path integral (10.1).

To obtain an intuition and to understand the local description, consider the example of the fuzzy sphere. We can solve for $X^{3}= \pm \sqrt{1-\left(X^{1}\right)^{2}-\left(X^{2}\right)^{2}}$, and use $X^{1}, X^{2}$ as local coordinate "near the north pole" $X^{3}=+1$ or the south pole $X^{3}=-1$. Each branch of the solution makes sense provided some restriction on the spectrum of $X^{3}$ is imposed, and in general "locality" might be phrased as a condition on the spectrum of some coordinate(s). Then the $X^{1}, X^{2}$ "locally" generate the full matrix algebra $\mathscr{A}$.

\footnotetext{
${ }^{9}$ It may happen that $\omega$ vanishes at certain locations, cf. [24]. This might be cured through compact extra dimensions.

${ }^{10}$ The use of the almost-Kähler structure may only be technical and should actually not be necessary.

${ }^{11}$ In topologically non-trivial situations they will individually generate only "almost" the full $\mathscr{A}$, and $\mathscr{A}$ is recovered by combining various such local descriptions. This will become more clear in the example of $S_{N}^{2}$.
} 
The splitting (7.1) can be refined using the $I S O(D)$ symmetry of Yang-Mills matrix models. In the semi-classical limit, one can assume (after a suitable rotation) that $\partial_{\mu} \phi^{i}=0$ for any given point $p \in \mathscr{M}$, so that the tangent space is spanned by the first $d$ coordinates in $\mathbb{R}^{D}$. Moreover, $p$ can be moved to the origin using the $D$-dimensional translations. Then the matrix geometry looks locally like $\mathbb{R}_{\theta}^{d}$, which is deformed geometrically by non-trivial $\phi^{i}\left(X^{\mu}\right)$ and a non-trivial commutator $\left[X^{\mu}, X^{v}\right]=i\left(\bar{\theta}^{\mu v}+\delta \theta^{\mu v}\left(X^{\alpha}\right)\right)$. These $X^{\mu} \sim x^{\mu}$ define "local embedding coordinates", which are analogous to Riemannian normal coordinates. Hence any deformation of $\mathbb{R}_{\theta}^{d}$ gives a matrix geometry as considered here, and vice versa any "locally smooth" matrix geometry should have such a local description. This justifies our treatment of matrix geometry.

\section{Generalized backgrounds}

Although we focused so far on matrix geometries which are quantizations of classical symplectic manifolds, matrix models are much richer and accommodate structures such as multiple branes, intersecting branes, manifolds suspended between branes, etc. For example, consider the following block-matrix configurations ${ }^{12}$

$$
Y^{a}=\left(\begin{array}{cccc}
X_{(1)}^{a} & 0 & 0 & 0 \\
0 & X_{(2)}^{a} & 0 & 0 \\
0 & 0 & \ddots & 0 \\
0 & 0 & 0 & X_{(n)}^{a}
\end{array}\right)
$$

Assuming that each block $X_{(i)}^{a}$ generates the (matrix) algebra $\mathscr{A}_{(i)}$ of functions on some quantized symplectic space $\mathscr{M}_{(i)} \subset \mathbb{R}^{D}$, it is clear that the matrices $Y^{a}$ should be interpreted as $n$ different NC branes embedded in $\mathbb{R}^{D}$. One way to see this is by considering optimally localized ("coherent", cf. section 3.6) states $|x\rangle_{(i)}$ corresponding to each block, such that the VEVs ${ }_{(i)}\left\langle x\left|X^{a}\right| x\right\rangle_{(i)} \approx x^{a}$ approximately sweep the location of $\mathscr{M}_{(i)} \subset \mathbb{R}^{D}$. Then clearly these state are also optimally localized for $Y^{a}$, and together sweep out the multiple brane configuration ${ }^{13}$. One particularly important case is given by a stack of $n$ coinciding branes:

$$
Y^{a}=X^{a} \otimes \mathbf{1}_{n}
$$

Fluctuations around such a background lead to $S U(n)$ Yang-Mills gauge theory on $\mathscr{M}$, as explained below. In fact, the underlying algebra $\mathscr{A} \otimes \operatorname{Mat}(\mathrm{n}, \mathbb{C})$ can be interpreted in two apparently different but nonetheless equivalent ways: 1$)$ as $s u(n)$ valued functions on $\mathscr{M}$ or 2) describing a higher-dimensional space $\mathscr{M} \times \mathscr{K}$, where $\operatorname{Mat}(\mathrm{n}, \mathbb{C})$ is interpreted as quantization of some compact symplectic space $\mathscr{K}$. Which of these two interpretations is physically correct depends on the actual matrix configuration, generalizing (8.2). Such extra dimensions allow to add more structure such as physically relevant gauge groups etc., cf. [28].

\footnotetext{
${ }^{12}$ Recall that by the Wedderburn theorem, the algebra generated by (finite-dimensional) hermitian matrices is always a product of simple matrix algebras, i.e. it decomposes into diagonal blocks as in (8.1).

${ }^{13}$ Elements of the off-diagonal blocks are naturally identified as bi-modules or "strings" connecting the branes. However we will not consider this here.
} 
Another interesting case if that of intersecting branes, such as two Moyal-Weyl quantum planes embedded along different directions. This is very useful to make contact with particle physics [30], but we only mention it here to illustrate the rich zoo of backgrounds of the matrix model, which is not restricted to smooth geometries.

\section{Effective gauge theory}

Consider a small deformation of the Moyal-Weyl quantum plane embedded in $\mathbb{R}^{D}$,

$$
X^{a}=\left(\begin{array}{c}
\bar{X}^{\mu} \\
0
\end{array}\right)+\left(\begin{array}{c}
-\bar{\theta}^{\mu v} A_{v} \\
\phi^{i}
\end{array}\right) .
$$

This can be interpreted either in terms of deformed geometry explained above, or in terms of NC gauge theory by considering $A_{\mu}=A_{\mu}(\bar{X})$ and $\phi^{i}=\phi^{i}(\bar{X})$ as gauge fields and scalar fields on $\mathbb{R}_{\theta}^{4}$. More precisely, fluctuations around a stack of branes (8.2) turn out to describe $\mathfrak{s u}(n)$-valued gauge fields coupled to the effective metric $G_{\mu \nu}$, and the matrix model (1.1) yields the effective action [5]

$$
S_{Y M}[A] \sim \frac{\Lambda_{0}^{4}}{4} \int d^{4} x e^{\sigma} \sqrt{\left|G_{\mu v}\right|} G^{\mu \mu^{\prime}} G^{\nu v^{\prime}} \operatorname{tr}\left(F_{\mu \nu} F_{\mu^{\prime} v^{\prime}}\right)+\frac{1}{2} \int \eta F \wedge F
$$

(dropping $\phi^{i}$ ). On $\mathbb{R}_{\theta}^{4}$, this is easy to understand: the gauge transformations $X^{a} \rightarrow U X^{a} U^{-1}$ give rise to

$$
A_{\mu} \rightarrow U A_{\mu} U^{-1}+i U \partial_{\mu} U^{-1}
$$

using (9.1), and the field strength $F_{\mu \nu}=\partial_{\mu} A_{\nu}-\partial_{v} A_{\mu}+i\left[A_{\mu}, A_{v}\right]$ is encoded in the commutator,

$$
\left[X^{\mu}, X^{v}\right]=-i \bar{\theta}^{\mu \mu^{\prime}} \bar{\theta}^{v v^{\prime}}\left(\bar{\theta}_{\mu^{\prime} v^{\prime}}^{-1}+F_{\mu^{\prime} v^{\prime}}\right) .
$$

Then (9.2) follows up to surface terms. However, the trace- $U(1)$ components of $A_{\mu}$ and $\phi^{i}$ should really be interpreted as embedding fluctuations of the brane, defining the effective metric $G_{\mu \nu}$ on a general $\mathscr{M} \subset \mathbb{R}^{D}$. Then the derivation of (9.2) becomes somewhat more technical, see [8, 29]. Nevertheless the gauge theory point of view is useful to carry out the quantization, as discussed below. This is a key feature of NC emergent gravity which greatly simplifies its quantization.

\section{Quantization and effective action}

Up to now, we discussed the geometry of some given NC space or brane in matrix models. It should be clear that such a quantum geometry does not amount to the quantization of a physical theory; it is a deformed classical geometry. To talk about quantum field theory or quantum gravity, we need to quantize the degrees of freedom in the model.

There are various ways of quantizing a classical theory: one can follow canonical quantization via a phase space formulation of the classical model, or attempt some sort of path-integral quantization. For matrix models, there is a very natural approach which is the analog of configuration-space path integral: Quantization is defined as an integration over the space of matrices. More explicitly, the partition function is defined as

$$
Z[J]=\int d X^{a} e^{-S[X]+X^{a} J_{a}},
$$


where we introduced external matrices $J^{a}$ in order to compute correlation functions and the effective action. The extension to fermions is straightforward. This definition respects the fundamental gauge symmetry $X^{a} \rightarrow U X^{a} U^{-1}$, as well as all global symmetries (translations, rotations, and possibly SUSY). These matrix integrals are known to exist ${ }^{14}$ for finite $N$.

Since the matrices $X^{a}$ encode both the geometry of the branes and also the propagating fields such as gauge fields, this matrix integral comprises quantum field theory as well as a quantum theory of geometry, hence of gravity. Moreover, there is no way to separate the field theoretical from the geometrical degrees of freedom. Hence we are facing a unified quantum theory of fundamental interactions including gravity, or some toy model thereof.

To obtain a potentially realistic model which describes near-classical geometries, the limit $N \rightarrow \infty$ of the quantization (10.1) must exist in some sense. This is of course highly non-trivial, and issues such UV-divergences, renormalization etc. arise in the $N \rightarrow \infty$ limit. In fact for most matrix models this limit probably does not make sense, or is very different from the semi-classical picture. However, there is essentially a unique model within this class of models (with $D \geq 4$ ) where this limit can be expected to exist, due to its maximal supersymmetry: the IKKT model [3]

$$
S_{\mathrm{IKKT}}=-(2 \pi)^{2} \operatorname{Tr}\left(\left[X^{a}, X^{b}\right]\left[X_{a}, X_{b}\right]+2 \bar{\Psi} \gamma_{a}\left[X^{a}, \Psi\right]\right),
$$

where $D=10$ and $\Psi$ are Majorana-Weyl spinors of $S O(9,1)$. On 4-dimensional NC brane backgrounds $\mathbb{R}_{\theta}^{4}$, this model can be viewed as $\mathscr{N}=4 \mathrm{NC}$ super-Yang-Mills gauge theory on $\mathbb{R}_{\theta}^{4}$, which is expected to be finite (at least perturbatively, but arguably also beyond) just like its commutative version. We will discuss some pertinent points below, and establish in particular one-loop finiteness.

A remark on the signature is in order. Majorana-Weyl spinors in $D=10$ exist only for Minkowski signature, transforming under the 10-dimensional Lorentz group $S O(9,1)$. This is of course the physically relevant case, and accordingly there should be an $i$ in the exponent in (10.1). However the Euclidean model does make sense e.g. after integrating out the fermions, and is mathematically easier to handle. We will therefore continue our discussion in the Euclidean case.

\subsection{UV/IR mixing in noncommutative gauge theory}

Using the gauge theory point of view, the quantization of matrix fluctuations in (9.1) can be carried out using standard field theory techniques adapted to the NC case. We need to quantize the gauge fields $A_{\mu}=A_{\mu}(\bar{X})$ and scalar fields $\phi^{i}=\phi^{i}(\bar{X})$ on $\mathbb{R}_{\theta}^{4}$. A general function on $\mathbb{R}_{\theta}^{4}$ can be expanded in a basis of plane waves, e.g.

$$
\phi(X)=\int \frac{d^{4} k}{(2 \pi)^{4}} \phi_{k} e^{i k_{\mu} \bar{X}^{\mu}} \in \operatorname{Mat}(\infty, \mathbb{C}) \cong \mathbb{R}_{\theta}^{4}
$$

where $\phi_{k}$ is an ordinary function of $k \in \mathbb{R}^{4}$. Inserting this into the action (1.1), the free (quadratic) part is then independent of $\theta^{\mu v}$, but the interaction vertices acquire a nontrivial phase factor $e^{\frac{i}{2} \sum_{i<j} k_{\mu}^{i} k_{\nu}^{j} \theta^{\mu \nu}}$ where $k_{\mu}^{i}$ denotes the incoming momenta. The matrix integral becomes an ordinary integral $\int d X^{a}=\int \Pi d \phi_{k}$, which can be evaluated perturbatively in terms of Gaussian integrals.

\footnotetext{
${ }^{14}$ more precisely, this has been established for the partition function and certain correlation functions [31].
} 
Thus Wicks theorem follows, however planar and non-planar contractions are distinct due to these phase factors, leading to Feynman-Filk rules. It turns out that planar diagrams coincide with their undeformed counterparts, while the non-planar diagrams involve oscillatory factors.

One can now compute correlation functions and loop contributions. In particular, the planar loop integrals have the same divergences as in the commutative case, in spite of the existence of a fundamental scale $\Lambda_{N C}$. This should not be too surprising, because noncommutativity leads to a quantization of area but not of length. However, a (virtual) UV momentum in some direction $k \gg \Lambda_{N C}$ necessarily implies a non-classical IR effect in another direction. This leads to the infamous UV/IR mixing [32], which technically originates from oscillatory integral due to the phase factors in non-planar diagrams. This phenomenon is ubiquitous in NC field theory, and leads to pathological IR divergences in any UV-divergent model, which cannot be cured by standard renormalization ${ }^{15}$.

As an illustration, we display the "strange" contribution of a scalar fields coupled to some external $U(1)$ gauge field in the 1-loop effective action:

$$
\begin{aligned}
\Gamma_{\Phi}= & -\frac{g^{2}}{2} \frac{1}{16 \pi^{2}} \int \frac{d^{4} p}{(2 \pi)^{4}}\left(-\frac{1}{6} F_{\mu v}(p) F_{\mu^{\prime} v^{\prime}}(-p) g^{\mu \mu^{\prime}} g^{v v^{\prime}} \log \left(\frac{\Lambda^{2}}{\Lambda_{\mathrm{eff}}^{2}}\right)\right. \\
& \left.+\frac{1}{4}(\theta F(p))(\theta F(-p))\left(\Lambda_{\mathrm{eff}}^{4}-\frac{1}{6} p \cdot p \Lambda_{\mathrm{eff}}^{2}+\frac{(p \cdot p)^{2}}{1800}\left(47-30 \log \left(\frac{p \cdot p}{\Lambda_{\mathrm{eff}}^{2}}\right)\right)\right)\right)
\end{aligned}
$$

where

$$
\frac{1}{\Lambda_{\mathrm{eff}}^{2}(p)}=\frac{1}{\Lambda^{2}}+\frac{1}{4} \frac{p^{2}}{\Lambda_{N C}^{4}} .
$$

is finite for $p \neq 0$ but diverges for $p \rightarrow 0$ and $\Lambda \rightarrow \infty$. These IR divergences become worse in higher loops, and the models are probably pathological as they stand.

For NC gauge theories as defined by matrix models, the geometrical insights explained above allows to understand this phenomenon in physical terms: Since fluctuations in the matrix model are understood as fields coupled to a non-trivial background metric, it follows that their quantization necessarily leads to induced gravity action, which diverge as $\Lambda \rightarrow \infty$. This is the standard mechanism of induced gravity due to Sakharov. This explanation of UV/IR mixing holds in the semi-classical regime i.e. for low enough cutoff, and has been verified in detail [21, 33] that these induced gravity terms give e.g. (10.4) in the appropriate limit.

We can now turn this problem into a virtue, noting that there is essentially one unique matrix model which does not have this problem (in 4 dimensions), given by the $\mathscr{N}=4$ SYM theory on $\mathbb{R}_{\theta}^{4}$, or equivalently the IKKT model (10.2). This is (almost) the unique model which is arguably well-defined and $U V$ finite to any order in perturbation theory, hence no such IR divergences arise ${ }^{16}$.

Accepting finiteness in the gauge theory point of view, it follows immediately from our geometrical discussions that the model provides a well-defined quantum theory of dynamical geometry in 4 dimensions, hence of (some type of) gravity. Moreover, there are clearly relations with general relativity: There are induced Einstein-Hilbert terms in the quantum effective action due to

\footnotetext{
${ }^{15}$ Renormalizable models do exist [34], at the expense of modifying the infrared behavior of the model e.g. through a confining potential. We refer to the contribution by $\mathrm{H}$. Grosse and M. Buric in this volume.

${ }^{16}$ These are not rigorous results at present but well justified by the relation with the commutative model [36], and partially verified by some loop computations in the NC case.
} 
(4.1), and moreover on-shell fluctuations of the would-be $U(1)$ gauge fields have been shown to be Ricci-flat metric fluctuations [6]. Nevertheless, at present there is no satisfactory understanding of the dynamics of this emergent gravity, due to the complexity of the system involving the Poisson structure. The presence of compactified extra dimensions can also be expected to play an important role here, and more work is needed to understand the effective gravity in this model.

\subsection{1-loop quantization of IKKT model}

In this last section, we illustrate the power and simplicity of the model by computing the full 1-loop effective action, and establish that it is UV finite on $\mathbb{R}_{\theta}^{4}$. The action induced by integrating out the fermions ${ }^{17}$ is given as usual by $\Gamma_{1-\text { loop }}^{(\Psi)}=-\frac{1}{4} \operatorname{Tr} \log \not D^{2}=-\frac{1}{4} \operatorname{Tr} \log \left(\square+\Sigma_{a b}^{(\psi)}\left[\Theta^{a b},.\right]\right)$, where

$$
\begin{aligned}
\left(\Sigma_{a b}^{(\psi)}\right)_{\beta}^{\alpha} & =\frac{i}{4}\left[\gamma_{a}, \gamma_{b}\right]_{\beta}^{\alpha} & & \text { fermions } \\
\left(\Sigma_{a b}^{(Y)}\right)_{d}^{c} & =i\left(\delta_{a}^{c} g_{b d}-\delta_{b}^{c} g_{a d}\right) & & \text { bosonic matrices }
\end{aligned}
$$

denote the generators of $S O(10)$ on the spinor and vector representations. To quantize the bosonic degrees of freedom is slightly more tricky due to the gauge invariance. We can use the background field method, splitting the matrices into background $X^{a}$ and a fluctuating part $Y^{a}$,

$$
X^{a} \rightarrow X^{a}+Y^{a} .
$$

For a given background $X^{a}$, the gauge symmetry becomes $Y^{a} \rightarrow Y^{a}+U\left[X^{a}+Y^{a}, U^{-1}\right]$, which we fix using the gauge-fixing function $G[Y]=i\left[X^{a}, Y_{a}\right]$. This can be done as usual using the FaddeevPopov method or alternatively using BRST [35]. Then the one-loop effective action induced by the bosonic matrices $Y^{a}$ is obtained as $\Gamma_{1-\text { loop }}^{(Y)}=\frac{1}{2} \operatorname{Tr} \log \left(\square+\Sigma_{r s}^{(Y)}\left[\Theta^{r s},.\right]\right)-\operatorname{Tr} \log (\square)$, where the last term is due to the FP ghosts. Hence the full contribution for the IKKT model is given by $[3,35]$

$$
\begin{aligned}
\Gamma_{\text {lloop }}[X] & =\frac{1}{2} \operatorname{Tr}\left(\log \left(\square+\Sigma_{a b}^{(Y)}\left[\Theta^{a b}, .\right]\right)-\frac{1}{2} \log \left(\square+\Sigma_{a b}^{(\psi)}\left[\Theta^{a b}, .\right]\right)-2 \log \square\right) \\
& =\frac{1}{2} \operatorname{Tr}\left(\log \left(\mathbf{1}+\Sigma_{a b}^{(Y)} \square^{-1}\left[\Theta^{a b}, .\right]\right)-\frac{1}{2}\left(\log \left(\mathbf{1}+\Sigma_{a b}^{(\psi)} \square^{-1}\left[\Theta^{a b}, .\right]\right)\right)\right. \\
& =\frac{1}{2} \operatorname{Tr}\left(-\frac{1}{4}\left(\Sigma_{a b}^{(Y)} \square^{-1}\left[\Theta^{a b}, .\right]\right)^{4}+\frac{1}{8}\left(\Sigma_{a b}^{(\psi)} \square^{-1}\left[\Theta^{a b}, .\right]\right)^{4}+\mathscr{O}\left(\square^{-1}\left[\Theta^{a b}, .\right]\right)^{5}\right) .
\end{aligned}
$$

The first 3 terms in this expansion cancel, which reflects the maximal supersymmetry. The traces are clearly UV convergent on 4-dimensional backgrounds such as $\mathbb{R}_{\theta}^{4}$, so that the 1-loop effective action is well-defined. Note that it incorporates both gauge fields and scalars, hence all gravitational degrees of freedom from the geometric point of view; for a more detailed discussion see [35]. Finiteness only holds for the IKKT model, while for all other models of this class with $D \neq 10$ already this 1-loop action is divergent. These divergences are in fact much more problematic than in the commutative case and cannot be handled with standard renormalization techniques, due to UV/IR mixing. Hence the NC case is much more selective than the commutative case, and the existence of an essentially unique well-behaved model is very remarkable.

\footnotetext{
${ }^{17}$ there is a subtlety - a Wess-Zumino contribution is missing here.
} 
Another remarkable aspect of this result is that the global $S O(10)$ invariance is manifestly preserved, and broken only spontaneously through the background brane such as $\mathbb{R}_{\theta}^{4}$. Such a statement would be out of reach within conventional quantum field theory, and noncommutativity is seen to provide remarkable new tools and insights.

At higher loops, perturbative finiteness is expected, because the UV divergences are essentially the same as in commutative $\mathscr{N}=4$ SYM [36]. Moreover UV/IR mixing results from the divergences at higher genus, which should also vanish by appealing to the large $N$ expansion of the commutative model. Alternatively, 1-loop finiteness along with $\mathscr{N}=1$ supersymmetry and the global $S O(10)$ or $S O(9,1)$ symmetry should ensure perturbative finiteness. These arguments remain to be made precise.

\section{Further aspects and perspectives}

Since the cosmological constant problem was raised in the introduction, we should briefly comment on this issue. Given our very limited understanding at present, no serious claims can be made. However, there are intriguing observations which raise the hope that this problem might be resolved here. The main point is that the metric is not a fundamental geometrical degrees of freedom, but a composite object which combines both the embedding of the brane $\mathscr{M}^{4} \subset \mathbb{R}^{D}$ and its Poisson tensor. This means that the equations of motion are fundamentally different from the Einstein equations even if the effective action has the standard Einstein-Hilbert form, and there will be new types of solutions which are less sensitive to the vacuum energy [5].

These different degrees of freedom are also the reason why the IKKT matrix model can be perturbatively finite, unlike general relativity. This model is much more suitable for quantization than GR. However, it remains to be shown that it also provides a physically viable description of gravity. There are several indications which suggest that this should be the case, including Ricciflat deformations on Moyal-Weyl space [6], the relation with IIB supergravity and string theory [3], the possibility to obtain Newtonian gravity [37], the fact that it gives some gravity theory with sufficiently rich class of geometries, etc.. However, the complicated interplay of the various degrees of freedom is not yet well understood, and more work is required before conclusions on the physical viability of this approach to quantum gravity can be drawn.

Finally a comparison with string theory is in order, since the IKKT model was proposed originally as a non-perturbative definition of IIB string theory. The link with IIB supergravity and string theory is established only for the interactions between brane solutions of the type we considered here. However, it may not reproduce e.g. all the massive degrees of freedom in string theory. Hence it seems more appropriate to consider the matrix model as a spin-off from string theory, which does provide a good quantum theory of 3+1-dimensional branes, but not necessarily for full string theory. In fact the 1-loop action is ill-defined e.g. on 8- and 10-dimensional branes, and the degrees of freedom of the metric are not fundamental but emergent and composite ${ }^{18}$. This in turn allows to avoid many problems of string theory, notably the lack of predictivity as illustrated in the landscape issue, while preserving many of its attractive features in a simpler framework.

In any case, this and related matrix models provide exciting new candidates for a quantum theory of gravity coupled to matter, and certainly deserve a thorough investigation.

\footnotetext{
${ }^{18}$ There is no problem with the Weinberg-Witten theorem which applies only for Lorentz-invariant field theories.
} 


\section{Acknowledgments}

I would like to thank the organizers of the $3^{\text {rd }}$ Quantum Gravity and Quantum Geometry School in Zakopane 2011 for providing a pleasant venue for stimulating and lively discussions with participants from various backgrounds. This work was supported by the Austrian Science Fund (FWF), project P21610.

\section{References}

[1] S. Doplicher, K. Fredenhagen, J. E. Roberts, “The Quantum structure of space-time at the Planck scale and quantum fields," Commun. Math. Phys. 172 (1995) 187-220. [hep-th/0303037].

[2] D. Amati, M. Ciafaloni, G. Veneziano, “Can Space-Time Be Probed Below the String Size?,” Phys. Lett. B216 (1989) 41.

[3] N. Ishibashi, H. Kawai, Y. Kitazawa and A. Tsuchiya, "A large-N reduced model as superstring," Nucl. Phys. B 498 (1997) 467 [arXiv:hep-th/9612115]

[4] T. Banks, W. Fischler, S. H. Shenker and L. Susskind, "M theory as a matrix model: A conjecture," Phys. Rev. D 55 (1997) 5112 [arXiv:hep-th/9610043].

[5] H. Steinacker, "Emergent Geometry and Gravity from Matrix Models: an Introduction," Class. Quant. Grav. 27 (2010) 133001 [arXiv:1003.4134 [hep-th]]; H. Steinacker, "Emergent Gravity and Noncommutative Branes from Yang-Mills Matrix Models," Nucl. Phys. B 810 (2009) 1 [arXiv:0806.2032].

[6] V. O. Rivelles, "Noncommutative field theories and gravity," Phys. Lett. B558 (2003) 191-196. [hep-th/0212262].

[7] H. S. Yang, "Exact Seiberg-Witten map and induced gravity from noncommutativity," Mod. Phys. Lett. A21 (2006) 2637-2647. [hep-th/0402002].

[8] H. Steinacker, "Emergent Gravity from Noncommutative Gauge Theory," JHEP 0712 (2007) 049. [arXiv:0708.2426];

[9] A. Connes, Publ. Math. IHES 62, 44 (1983); Noncommutative Geometry (Academic Press, New York 1994).

[10] M. Bordemann, E. Meinrenken and M. Schlichenmaier, "Toeplitz quantization of Kahler manifolds and gl(N), N $\rightarrow$ infinity limits," Commun. Math. Phys. 165 (1994) 281 [arXiv:hep-th/9309134]; S. Twareque Ali, M. Englis, "Quantization Methods: A Guide for Physicists and Analysts". Rev. Math. Phys. 17 (2005) 391-490; [arXiv:math-ph/0405065]; S. Waldmann, "Poisson-Geometrie und Deformations-quantisierung”. Springer, 2007

[11] M. Kontsevich, “Deformation quantization of Poisson manifolds, I,” Lett. Math. Phys. 66 (2003) 157 [arXiv:q-alg/9709040].

[12] J. Madore, “The Fuzzy sphere,” Class. Quant. Grav. 9 (1992) 69-88.

[13] J. Hoppe, "Quantum theory of a massless relativistic surface and a two-dimensional bound state problem“, PH D thesis, MIT 1982; J. Hoppe,"Membranes and matrix models," [hep-th/0206192].

[14] M. Chaichian, A. Demichev and P. Presnajder, "Quantum field theory on noncommutative space-times and the persistence of ultraviolet divergences," Nucl. Phys. B 567 (2000) 360 [arXiv:hep-th/9812180].

[15] A. M. Perelomov, "Generalized coherent states and their applications," Berlin, Springer (1986) 
[16] H. Grosse, P. Presnajder, "The Construction of noncommutative manifolds using coherent states," Lett. Math. Phys. 28 (1993) 239-250.

[17] H. Grosse, J. Madore, H. Steinacker, "Field theory on the q deformed fuzzy sphere. 1.," J. Geom. Phys. 38 (2001) 308-342. [hep-th/0005273].

[18] A. Connes, M. R. Douglas, A. S. Schwarz, "Noncommutative geometry and matrix theory: Compactification on tori," JHEP 9802 (1998) 003. [hep-th/9711162].

[19] P. B. Gilkey, "Invariance theory, the heat equation and the Atiyah-Singer index theorem,". CRC Press, 1995.

[20] A. Connes, "Gravity coupled with matter and the foundation of non-commutative geometry," Commun. Math. Phys. 182 (1996) 155 [arXiv:hep-th/9603053]; A. Connes, "A unitary invariant in Riemannian geometry,” Int. J. Geom. Meth. Mod. Phys. 5 (2008) 1215 [arXiv:0810.2091].

[21] D. N. Blaschke, H. Steinacker, M. Wohlgenannt, "Heat kernel expansion and induced action for the matrix model Dirac operator," [arXiv:1012.4344].

[22] D. N. Blaschke, H. Steinacker, "Curvature and Gravity Actions for Matrix Models," Class. Quant. Grav. 27 (2010) 165010. [arXiv:1003.4132]; D. N. Blaschke, H. Steinacker, "Curvature and Gravity Actions for Matrix Models II: The Case of general Poisson structure,’ [arXiv:1007.2729].

[23] J. Arnlind, J. Hoppe, G. Huisken, "Discrete curvature and the Gauss-Bonnet theorem," [arXiv:1001.2223].

[24] D. N. Blaschke, H. Steinacker, "Schwarzschild Geometry Emerging from Matrix Models," Class. Quant. Grav. 27 (2010) 185020. [arXiv:1005.0499].

[25] C.J.S. Clarke, “On the Global Isometric Embedding of Pseudo-Riemannian Manifolds”, Proc. Royal Soc. London, A 314, 417 (1970); A. Friedman, J. Math. Mech. 10, 625 (1961)

[26] N. Kuntner, H. Steinacker, “On Poisson geometries related to noncommutative emergent gravity” in preparation.

[27] D. Borthwick, A. Uribe, "Almost complex structures and geometric quantization“. Math. Res. Letters 3, 845-861 (1996); [arXiv:dg-ga/9608006]

[28] P. Aschieri, T. Grammatikopoulos, H. Steinacker and G. Zoupanos, "Dynamical generation of fuzzy extra dimensions, dimensional reduction and symmetry breaking," JHEP 0609 (2006) 026 [arXiv:hep-th/0606021]; H. Grosse, F. Lizzi and H. Steinacker, "Noncommutative gauge theory and symmetry breaking in matrix models,” Phys. Rev. D 81 (2010) 085034 [arXiv:1001.2703].

[29] H. Steinacker, "Covariant Field Equations, Gauge Fields and Conservation Laws from Yang-Mills Matrix Models,” JHEP 0902 (2009) 044. [arXiv:0812.3761].

[30] A. Chatzistavrakidis, H. Steinacker, G. Zoupanos, "Intersecting branes and a standard model realization in matrix models," [arXiv:1107.0265].

[31] P. Austing, J. F. Wheater, “Convergent Yang-Mills matrix theories,” JHEP 0104 (2001) 019. [hep-th/0103159]; W. Krauth, M. Staudacher, "Finite Yang-Mills integrals,” Phys. Lett. B435 (1998) 350-355. [hep-th/9804199].

[32] S. Minwalla, M. Van Raamsdonk, N. Seiberg, "Noncommutative perturbative dynamics," JHEP 0002 (2000) 020. [hep-th/9912072].

[33] H. Grosse, H. Steinacker, M. Wohlgenannt, "Emergent Gravity, Matrix Models and UV/IR Mixing," JHEP 0804 (2008) 023. [arXiv:0802.0973 [hep-th]]. 
[34] H. Grosse, R. Wulkenhaar, "Renormalization of phi**4 theory on noncommutative $\mathrm{R} * * 4$ in the matrix base," Commun. Math. Phys. 256 (2005) 305-374. [hep-th/0401128].

[35] D. N. Blaschke, H. Steinacker, "On the 1-loop effective action for the IKKT model and non-commutative branes," [arXiv:1109.3097].

[36] I. Jack, D. R. T. Jones, “Ultraviolet finiteness in noncommutative supersymmetric theories," New J. Phys. 3 (2001) 19. [hep-th/0109195].

[37] H. Steinacker, "On the Newtonian limit of emergent NC gravity and long-distance corrections," JHEP 0912 (2009) 024. [arXiv:0909.4621 [hep-th]]. 